\title{
Medicina Interna: esperienze e progettualità per una forte integrazione con i bacini di riferimento territoriale
}

\author{
Internal Medicine: experience and planning for close integration \\ with territorial reference points
}

\section{Introduzione}

Il presente articolo riporta l'intervento dell'autore alla tavola rotonda "Medicina Interna, patologie croniche, paziente fragile, dimissioni protette" tenutasi nell'ambito del Convegno FADOI Medicina Interna oggi tra nuovi bisogni, nuovi modelli e nuove risposte: a che punto siamo? (Bologna, 23 settembre 2010).

Il tema del ruolo delle Medicine Interne ospedaliere nell'epoca delle "grandi fragilità" viene affrontato non in termini meramente teorici, ma dal punto di vista della direzione di un'azienda sanitaria territoriale, a bacino d'utenza provinciale, che ha in diretta gestione:

- un ospedale in zona montana (Ospedale Santa Maria di Borgo Val di Taro), che ha percorso un'esperienza di forte integrazione con il complesso dei servizi sanitari che forniscono risposta ai bisogni epidemiologicamente più rilevanti del territorio di riferimento;

- un ospedale di più ampie dimensioni (Ospedale di FidenzaSan Secondo Parmense), con tre Dipartimenti (Medico, Chirurgico e di Emergenza-Urgenza), caratterizzato da eccellenze in alcune discipline medico-chirurgiche e fortemente integrato nella rete "hub and spoke" provinciale e regionale. Un ospedale, dunque, in grado di fornire risposte ai bisogni di salute di una popolazione di un'area più vasta e capace di costituire un polo di attrazione per zone limitrofe.

\section{L'esperienza e la progettualità della Medicina Interna dell'ospedale montano}

\section{La strutturazione attuale}

Nell'Ospedale Santa Maria di Borgo Val di Taro, l'Unità Operativa Complessa (UOC) di Medicina Interna è inserita nel
Dipartimento di Medicina e Diagnostica, che può disporre delle seguenti strutture di degenza:

1) una funzione di terapia subintensiva cardiologica (3 posti letto);

2) una funzione di riabilitazione (intensiva) cardiologica (6 posti letto);

3) una funzione di lungodegenza post-acuzie (16 posti letto);

4) posti letto di Medicina Interna per Acuti, compresa l'Osservazione Breve del Punto di Primo Intervento Ospedaliero;

5) un day hospital oncologico (7 posti letto).

Le prime quattro funzioni sono collocate sullo stesso piano, mentre il day hospital è collocato in area distinta, con ingresso proprio.

Allo stesso piano delle funzioni da 1 a 4 è presente un hospice territoriale, dotato di 8 posti letto, dei quali 2 destinati alle gravi disabilità acquisite in età adulta, gestito in perfetta sinergia con l'assistenza domiciliare distrettuale e a direzione infermieristica.

La direzione dell'UOC di Medicina Interna dell'Ospedale Santa Maria di Borgo Val di Taro coincide con quella del Dipartimento di Medicina e Diagnostica e si correla fortemente con la direzione del Dipartimento delle Cure Primarie del Distretto delle Valli del Taro e del Ceno, che comprende i territori della vasta zona appenninica posta a ovest della provincia di Parma.

Le competenze specialistiche presenti nell'organico dell'UOC di Medicina Interna coprono la gran parte delle esigenze di maggior impatto epidemiologico:

- cardiologia e riabilitazione cardiologica;

- ipertensivologia;

- diabetologia;

- geriatria e cure palliative;

- nefrologia;

- pneumologia (di derivazione aziendale). 
La direzione del Dipartimento di Medicina e Diagnostica si inserisce in un'organizzazione complessiva di ospedale/distretto che vede al vertice gestionale il direttore del Distretto, il quale risulta quindi il garante della corrispondenza delle esigenze della committenza con gli orientamenti della produzione.

Nei percorsi diagnostico-terapeutici integrati, un ruolo fondamentale di raccordo è attribuito alla professione infermieristica. In particolare, tre funzioni sono determinanti per costruire le sinergie tra ospedale e territorio:

- la lungodegenza post-acuzie;

- l'hospice;

- l'assistenza domiciliare.

$\dot{E}$ in via di attuazione un progetto specifico di integrazione "forte" delle tre funzioni, attraverso l'individuazione di una comune direzione infermieristica.

\section{Il progetto di miglioramento della continuità assistenziale}

\section{Il contesto di riferimento}

Il sistema sanitario delle Valli del Taro e del Ceno si caratterizza da tempo per la messa a punto di servizi ospedalieri e territoriali in rete, secondo criteri di continuità e gradualità delle cure. In tal senso, a partire dall'anno 1999, nell'Ospedale Santa Maria è stata realizzata la Lungodegenza PostAcuzie, in contiguità con l'UO di Medicina Interna, mentre nel Dipartimento delle Cure Primarie del Distretto delle Valli del Taro e del Ceno è stato aperto, nell'anno 2005, l'Hospice "La Valle del Sole". In parallelo con le due precedenti realizzazioni, sono stati potenziati i servizi infermieristici domiciliari. L'avvio di tali nuovi servizi è stato accompagnato da esperienze gestionali innovative, quali la direzione infermieristica dell'hospice.

La pratica di protocolli condivisi per la gestione delle dimissioni protette, dall'ospedale al domicilio, e l'approccio interdisciplinare, assunto come modalità operativa comune a tutti i percorsi ospedale-territorio, hanno costituito le novità organizzative più rilevanti. Ne sono testimonianza l'integrazione del personale infermieristico nelle attività assistenziali domiciliari e presso l'hospice, e l'interazione tra infermieri, medici palliativisti e psicologi nell'attività dell'hospice e della lungodegenza.

\section{L'obiettivo del progetto}

Con il progetto si vuole sviluppare ulteriormente l'assistenza, secondo criteri di continuità e gradualità delle cure, creando condizioni organizzative di maggiore strutturazione dei percorsi integrati tra l'ospedale e il territorio.

In particolare, si intende costruire un collegamento strutturato tra il Dipartimento di Medicina e Diagnostica dell'Ospedale e il Dipartimento delle Cure Primarie del Distretto, all'interno dei quali si svolge il percorso del paziente, dalla diagnosi e dall'impostazione terapeutica in acuzie (UO di Medicina Interna e Struttura Semplice di Cardiologia) alla stabilizzazione in post-acuzie (lungodegenza), alla dimissione protetta verso il domicilio, con ritorno al medico di famiglia e all'équipe territoriale domiciliare e, nei casi che lo richiedono, all'hospice.

La sperimentazione di tali percorsi assistenziali, per la quale si prevede un primo periodo della durata di circa un anno, potrà essere utile anche per rivisitare, in una fase successiva, i percorsi di gestione e dimissione del paziente operato e ricoverato nei posti letto di riabilitazione estensiva ortopedica.

\section{Azioni previste dal progetto}

Si prevedono azioni strutturali, organizzative e formative.

Azioni strutturali. Si prevede di rimodulare i posti letto della lungodegenza secondo criteri di maggiore accoglienza e comfort, con una loro collocazione sempre contigua, ma chiaramente distinta e separata, rispetto ai posti letto per acuti della Medicina Interna, a sottolineare il doppio carattere di gradualità (la differenziazione) e di continuità (la contiguità fisica) tra i due livelli assistenziali.

Azioni organizzative e attività formative di supporto. $\mathrm{Si}$ prevede l'individuazione di una "funzione di raccordo assistenziale unico" tra il Dipartimento di Medicina, cioè tra le aree assistenziali di Medicina e di Lungodegenza dell'Ospedale, e le strutture distrettuali dell'hospice e delle cure domiciliari, per conferire continuità al percorso di assistenza.

Tale funzione di raccordo assistenziale risponde ai seguenti criteri:

- è sovraordinata ai coordinamenti infermieristici di UO previsti dalla programmazione aziendale;

- risponde al direttore del Dipartimento di Medicina e al direttore del Dipartimento delle Cure Primarie, per le rispettive competenze;

- sovrintende all'attività assistenziale delle articolazioni organizzative sopra delineate;

- assicura la fluidità dei percorsi assistenziali e l'integrazione operativa tra gli operatori assegnati alle diverse articolazioni strutturali/organizzative;

- promuove la formazione integrata del personale;

- sovrintende ai percorsi di protezione delle dimissioni difficili.

Si prevede altresì l'acquisizione di una funzione di supporto psicologico, in grado di presidiare tutto il percorso di cura, dalla Medicina Interna alla lungodegenza, all'hospice.

Tale funzione non sostituisce con l'attività di supporto psicologico già in essere presso l'hospice, ma si integra con essa ed è alle dipendenze del direttore del Dipartimento di Medicina.

Si stabilisce di inserire il tema della continuità dei percorsi di cura tra le priorità del Piano delle Attività formative 2010 del Presidio Ospedaliero Aziendale, assegnando particolare rilievo alle iniziative programmate in comune dall'Ospedale Santa Maria e dal Distretto delle Valli del Taro e del Ceno.

\section{Risorse aggiuntive previste per il progetto e tempi di realizzazione}

È previsto il finanziamento di un contratto libero professionale per 18 ore settimanali con uno psicologo.

È prevista l'assegnazione temporanea della funzione di raccordo assistenziale sopra descritta a un infermiere titolare di Posizione Organizzativa, con esperienza documentata in materia ospedaliera e territoriale, con particolare riferimento al presidio di percorsi di continuità delle cure.

La fase sperimentale del progetto è stata quantificata nel periodo intercorrente dall'1 dicembre 2009 al 31 dicembre 2010. 
Entro 60 giorni dall'avvio della sperimentazione, l'infermiere coordinatore incaricato del raccordo assistenziale deve produrre una prima relazione, evidenziante punti di forza e criticità dell'attuale organizzazione, con prime proposte di miglioramento.

Una verifica in itinere del progetto è stata altresì programmata entro il primo semestre del 2010.

\section{Il contesto e la progettualità innovativa dell'ospedale di più ampie dimensioni e complessità}

\section{Il contesto attuale}

Nell'Ospedale di Fidenza-San Secondo Parmense, molte discipline mediche assumono la connotazione di Strutture Complesse (SC; per esempio Pronto soccorso e Medicina d'Urgenza, Cardiologia, Urologia, Neurologia, Pediatria), mentre altre funzioni sono organizzate come Strutture Semplici (SS), con forte connotazione di autonomia pur nell'ambito dell'afferenza a Strutture Complesse.

In ambito internistico, alle due UOC di Medicina Interna afferiscono le SS di Oncoematologia, Nutrizione Artificiale Domiciliare, Fisiopatologia Respiratoria, Emodialisi, Diabetologia ed Emostasi.

La Medicina Interna è inserita nel Dipartimento di Medicina Generale Specialistica e Riabilitativa, cui afferisce pure la Lungodegenza Post-Acuzie e Riabilitazione Estensiva, collocata fisicamente in un altro stabilimento ospedaliero ubicato a circa $35 \mathrm{~km}$ di distanza.

\section{Il progetto per la riorganizzazione della Medicina Interna di Fidenza per intensità di cura}

Nel piano delle azioni aziendali è inserita la proposta di riorganizzazione delle UO di Medicina Interna per intensità di cure.

Il nuovo modello si basa su posti letto funzionalmente caratterizzati dal consumo di risorse omogenee ma determinate in base a diversi bisogni assistenziali. Le attività ospedaliere si svolgono in aree differenziate per modalità assistenziali, intensità delle cure, durata della degenza e regime di ricovero.

Questo nuovo modello permette di porre il paziente al centro del processo di cura con carichi assistenziali modulati secondo le necessità oggettive; i professionisti sono impiegati in più settori di cura con diverse fisionomie (tutor, specialist, consultant) a seconda delle esigenze del caso, e i diversi settori specialistici vedono le barriere ridursi fino a scomparire (superamento del livello organizzativo per reparti).

In generale $\mathrm{i}$ livelli di cure previsti sono tre:

- intensivo e semintensivo: alta intensità di cura - letti intensivi o subintensivi;

- acuto: media intensità di cura - letti di degenza ordinaria;

- post-acuto: bassa intensità di cura - letti di lungodegenza e riabilitazione.

L'esigenza della suddivisione deriva dalla specifica tipologia dei pazienti che vengono ricoverati nei reparti di Medicina Interna, caratterizzati da estrema variabilità del quadro clinico e del relativo carico assistenziale, per evitare di erogare a tutti prestazioni simili, che possono risultare superiori alle necessità per alcuni pazienti e insufficienti per altri. La prima situazione si verifica quando un utente con elevati bisogni viene inserito in un sistema a bassa offerta: come conseguenza il sistema si stressa e il paziente può ottenere più assistenza rispetto agli altri ricoverati, ma senza ricevere quella di cui necessita. La seconda situazione si realizza se l'utente con modesti bisogni è inserito in un sistema ad alta offerta, per cui riceve una quota assistenziale superiore a quella necessaria, in termini sia qualitativi sia quantitativi. Quando questi effetti coesistono, se ne sommano i rischi. Si avverte, allora, la necessità di riorganizzare l'assistenza ponendo al centro il paziente e i suoi bisogni in quella fase specifica del suo decorso, identificando aree di degenza omogenee per livelli di cura e proponendo livelli di attenzione clinica e indicatori di complessità assistenziale simili nella medesima area.

L'accoglienza del paziente in un'area di degenza appropriata per il suo livello di cura deve attenersi a criteri oggettivi che fanno riferimento a due parametri: l'instabilità (severità clinica) e la complessità assistenziale. Occorre considerare parametri e scale che tengano conto sia dell'instabilità del paziente dal punto di vista medico, sia della complessità assistenziale infermieristica (per esempio, la scala di Glasgow per lo stato generale, la scala di Child-Pugh per la gravità delle epatopatie, la scala del British Medical Council per il grado di dispnea, le scale di Norton plus e di Braden per i decubiti, le scale del dolore ecc.). Nella dimensione dell'UO di Medicina Interna, l'organizzazione per livelli di cura si traduce in un più appropriato grado di attenzione al paziente e alla sua patologia tramite il cosiddetto "triage di corridoio", messo in atto, quando il paziente giunge in reparto, da un team adeguatamente istruito e composto dal medico di turno e dall'infermiere di riferimento.

Il modello proposto ridisegna il modello organizzativo ospedaliero tramite l'accorpamento di Unità Operative, già strettamente relate sul piano clinico, che condividono risorse umane e conoscenze scientifiche.

Tale tipo di riorganizzazione presenta ovviamente notevoli difficoltà attuative, soprattutto laddove si debbano realizzare, fra i professionisti, integrazione e coordinamento dei processi di cura, gestione in comune di alcune risorse, in particolare dei posti letto, snellimento organizzativo, recupero della centralità del paziente (con il superamento della logica secondo la quale il paziente è proprietà dell'Unità Operativa), preservazione della specializzazione, grazie alla creazione di gruppi di studio-lavoro che affrontino specifici problemi di salute.

Un elemento particolarmente cruciale è la necessità di gestire il malato post-acuto, che rappresenta uno snodo fondamentale nelle competenze e nelle attività della moderna Medicina Interna. Infatti, mentre si assiste all'allungamento della vita media della popolazione e, di conseguenza, alla maggiore sopravvivenza del malato cronico polipatologico, tipico dei reparti internistici, la politica sanitaria degli ultimi decenni identifica la funzione dell'ospedale come struttura ad alto impatto economico nella gestione dei pazienti acuti o cronici riacutizzati, introducendo i concetti di appropriatezza del ricovero, di efficienza e di corretta allocazione delle risorse, e inducendo la strategia comune di riduzione complessiva dei posti letto e di contrazione delle 
giornate di degenza. L'ospedale, per contro, viene inteso come il luogo più appropriato per la gestione dei problemi di salute, più o meno acuti, e per le situazioni socioambientali di tipo non strettamente medico.

La Struttura di Medicina Interna è la sede in cui si concentrano, oltre a problemi medici, anche altre problematiche (sociali) non suscettibili di immediata risoluzione. L'età risulta fattore condizionante per l'inappropriatezza del ricovero in area per acuti. In una struttura per pazienti postacuti, attigua, funzionalmente e strutturalmente, alla degenza ordinaria di Medicina Interna, può essere ricoverata una popolazione ben definita costituita da grandi anziani che, dopo l'intervento acuto, presentano difficoltà di stabilizzazione e di ripresa e richiedono più tempo di ricovero e attivazione di cure intermedie. Tale struttura protetta e specializzata potrebbe favorire la stabilizzazione clinica e permettere un più precoce recupero di autonomia, in attesa della collocazione nell'ambiente più idoneo alla dimissione. In un'ottica di allocazione di risorse limitate, l'istituzione di aree specializzate per malati post-acuti potrebbe consentire di migliorare la gestione della patologia e dell'invalidità di una popolazione di grandi anziani. Rispetto al ricovero ordinario, tale struttura organizzativa potrebbe comportare una riduzione di spesa, consentire una maggiore autonomia alle dimissioni e un minore ricorso all'istituzionalizzazione protratta con i rischi connessi (infettivi in primis).

\section{Conclusioni}

La tipologia dei nostri ospedali provinciali rappresenta appieno le esigenze di due territori diversificati per bisogni e popolazioni e realizza la più grande integrazione fra ospedale e territorio. I reparti di Medicina Interna sono l'espressione più solida di tale integrazione.

Il piccolo Ospedale di Borgo Val di Taro (120 posti letto), strutturato e organizzato per fornire risposte a una popolazione residente in valli montane lontane da centri di alto livello, è non solo un'azienda importante per l'economia locale, ma anche una risorsa di salute imprescindibile. Mentre le strutture chirurgiche si basano prevalentemente su lavoro programmato, il Dipartimento di Medicina Interna, in questo contesto, costituisce il principale punto di riferimento e il capofila dell'integrazione con il territorio, perché, con le sue articolazioni - punto di primo intervento, ricovero, lungodegenza - riesce a farsi carico dei bisogni urgenti e di continuità di cura soprattutto degli anziani, fragili, complessi, che rappresentano la maggior parte dei pazienti che accedono al nosocomio.

L'Ospedale di medie dimensioni di Fidenza (250 posti letto) rappresenta probabilmente la tipologia ottimale per raggiungere obiettivi di quantità e di qualità delle prestazioni sanitarie, per una struttura di livello provinciale. Gli obiettivi quantitativi rispondono all'esigenza di fornire risposte a un bacino di utenza più ampio, contrastando i fenomeni di mobilità passiva e, ove possibile, inducendo fenomeni di mobilità attiva. Gli obiettivi qualitativi attengono alla capacità di utilizzare, mediante l'impiego di elevata professionalità, tecnologie di alto livello che consentano di integrarsi, oltre che con il territorio, con le strutture hub di riferimento. In questo contesto la Medicina Interna e la nuova organizzazione per intensità di cure rappresentano l'elemento gestionale innovativo che può consentire di porre il paziente al centro del processo di cure, di razionalizzare le risorse e di promuovere l'autonomia professionale del personale sanitario medico e infermieristico. Lo sviluppo della Medicina Interna come specializzazione della complessità è un elemento da sostenere e valorizzare da parte di un'azienda sanitaria territoriale.

\section{Bibliografia di riferimento}

Alessandri M, Bartolomei C, Bernardini M, Landini G, Laureano R, Lombardo $G$, et al. Medicina interna e nuova organizzazione ospedaliera: la proposta della FADOI Toscana. It J Med 2007;1(1):65-9.

Deliberazione Giunta Regionale dell'Emilia-Romagna, 8 febbraio 2010, n. 291. Casa della Salute: indicazioni regionali per la realizzazione e l'organizzazione funzionale. 1.1. Modelli assistenziali e strategie regionali per le cure territoriali. Regione Emilia-Romagna, Bollettino Ufficiale 18 marzo 2010.

Tiratterra F, Pignatelli V, Placido A, Somma G, Pietroiusti A. Comorbility and recurrent hospitalization in Internal Medicine. G Gerontol 2008;56:187-90.

\author{
Massimo Fabi \\ Direttore generale \\ Azienda USL di Parma \\ E-mail: mfabi@ausl.pr.it
}

